Natalie Pennington* and Jeffrey A. Hall

\title{
An analysis of humor orientation on Facebook: A lens model approach
}

\begin{abstract}
This article presents the results of a mixed method analysis of the use and perception of humor orientation (HO) on Facebook (FB) profiles $(N=100)$. Results of the lens model analysis suggest that a variety of profile cues, not just those directly related to humor, are used by FB users to demonstrate $\mathrm{HO}$ and by observers to perceive HO. Cues used by profile owners and perceived by strangers as indicative of a humorous disposition include: status updates that contained relational talk, humor in profile pictures, humor in quotes, the number of times FB friends "liked" status updates, and the number of unique friends who commented on status updates. Additionally, political talk in status updates was negatively related to users' HO and observers' impressions of users' HO. A qualitative thematic analysis of the FB profiles was then conducted. Those analyses suggested that cues diagnostic of users' HO thematically focused on daily life events, pop-culture references, and self-related anecdotes. Implications for the expression of and perception of humor on FB are discussed.
\end{abstract}

Keywords: Facebook, humor, lens model, online impression management

*Corresponding author: Natalie Pennington: Communication Studies Department, University of Kansas, Bailey Hall, 1440 Jayhawk Blvd. Rm 102, Lawrence, KS 66045. E-mail: natpen@ku.edu Jeffrey A. Hall: University of Kansas. E-mail: hallj@ku.edu

\section{Rationale and literature review}

Given the uncertainty that can arise when communicating online, there is a natural desire to engage in humor (Baym 1995). By sharing a joke with others, we can build common ground and reduce uncertainty when we are unable to be copresent (Rains et al. 2009). The ability to convey humor online is a dynamic process that can be navigated through multiple media, including fan forums (Baym 1995), dating websites (Wilbur and Campbell 2011), and social networking sites (SNS) (Carr et al. 2012). Each medium provides the opportunity for a user to manage their identity with an online audience. A popular and developing form of mediated self-presentation can be found on Facebook (FB), where 800 million users $\log$ on daily to share information about their lives with friends (Facebook 
Statistics 2011). From status updates to pictures to interests, FB offers users multiple ways to intentionally construct and manage their identity online (Zywica and Danowski 2008).

The present research is concerned with two primary goals: (i) how individuals use features of FB to display humor, and (ii) what features of FB are perceived by strangers to be indicative of users' humor orientation (HO). To accomplish these aims, this study utilizes a mixed method approach. A lens model analysis of humor on FB will illuminate what specific cues on a FB profile are related to profile owners' $\mathrm{HO}$, and what cues are used by strangers to estimate HO in a profile owner (PO). The lens model also allows researchers to identify diagnostic cues (Gifford 2006), which are cues used by POs and perceived by strangers to estimate HO. An indepth qualitative analysis of FB profiles will identify themes of humorous communication on FB. By combining quantitative and qualitative analysis of a single data set, this project offers a detailed and holistic study of how humor is produced and perceived on FB. The primary contribution of the present investigation is to showcase how humor is perceived (and actually used) on the popular SNS, Facebook.

\subsection{Humor and social desirability}

Humor can be understood as the use of "intentional verbal or nonverbal messages, which elicit laughter, chuckling, and other forms of spontaneous behavior taken to mean pleasure, delight, and/or surprise in the targeted receiver" (BoothButterfield and Booth-Butterfield 1991: 206). Knowing how to produce humor, defined as humor orientation (HO), is considered one of the most desirable traits a person can have (Cann et al. 1997). To charm a potential romantic partner with an anecdote or share jokes among friends, we value humor not only in ourselves, but in the company we keep. Research suggests the desirability of humor stems from a belief that humor makes a person socially competent, and as a result, well liked by peers (Martin 1998). This perception is backed by research that humor is related to social competence (Wanzer et al. 1996). A high degree of HO is associated with conversational sensitivity and nonverbal sensitivity, which suggests that high HO individuals can develop strong interpersonal relationships (Merolla 2006). But how do we go about expressing humor online?

\subsection{Humor online}

Impression management is any behavior an individual engages in that demonstrates to the audience particular characteristics of the self (Goffman 1959). These 
performances occur in the presence of others and vary in the degree to which the individual acts intentionally and consciously. Walther's (1996) hyperpersonal model suggests that in a computer-mediated communication (CMC) context, a communicator is afforded opportunities to manipulate self-presentation that would not be possible in face-to-face encounters. As a consequence, online selfpresentations are more carefully crafted and intentional than offline (Zywica and Danowski 2008). The hyperpersonal model suggests that while individuals act strategically to convey a desirable impression of self, these self-presentations are constrained by the communication medium itself. Despite the limitations of CMC, past research (e.g., Baym 1995; Nastri et al. 2006) has demonstrated that being perceived as humorous is a common goal online.

Baym's (1995) research on humor within a text-based fan forum claims that humor expressed online has the ability to establish individuality due to its performative nature. The act of producing humor (e.g., carefully crafting a joke; poking fun at a discussion topic) increased attention on the individual posting. Other users' responses to that humorous act further influenced how the online community viewed that individual. Although the response of others is an important part of humor online or offline, it was amplified in CMC because humor can affect how others perceive the personality of someone with whom they are unacquainted offline. Therefore, humorous posts are perceived as highly enjoyable and receive the most attention from readers (Baym 1995).

Even online messages sent to a generalized but unknown CMC audience are capable of conveying humor. In an analysis of away messages within instant messaging (IM), Nastri et al. (2006) found that users posted messages that were both informative and entertaining. An away message is created so that if a user is away from their computer but still logged into chat, other IM users know what the user is doing. Although the recipient of the away message is likely known to the IM user, the message is crafted for a generalized other. Nastri et al. (2006) found that one-fifth of all away messages contained humor and wit, which suggests that humor is a common form of self-expression in this form of CMC.

Most email servers provide the user with an electronic signature option, which can be see as similar to an away message. Rains et al. (2009) found that the second most frequent category used for signatures was humor. Rains et al. provided the following as some of their examples: "I have never taken an exercise except sleeping and resting" and "When you get upset, remember it takes 42 muscles to frown, and only 4 to extend your middle finger!” (113). Email lists (Hübler and Bell 2003) have also been analyzed for humor, with researchers finding that humor was produced to create shared meaning and identity among members.

These studies show that humor is frequently enacted as a tool of impression management within CMC. Missing from the literature is an analysis of how HO is 
conveyed in SNS. Although Carr et al. (2012) analyzed status updates on FB as speech acts, these statements were considered in isolation from the message creator and impressions of others. A comprehensive analysis of how humor is employed on FB to account for both impression management and impression formation is needed.

\subsection{Impression management on Facebook}

One primary feature of FB is creating a profile. As Zywica and Danowski (2008) point out, creating a profile is a deliberate construction of self. The profile provides the opportunity to display personality, particularly to emphasize traits that are believed to be desirable to a users' audience. The FB profile contains a variety of cues that can be used to ascertain the PO's personality (Utz 2010; Walther et al. 2009). These cues can be self-generated (i.e., information provided by the PO), other-generated (i.e., information provided by the friends of the PO), or systemgenerated (i.e., information reported by FB itself) (Utz 2010). Some examples of self-generated cues include the profile picture, status updates, and likes and interests. Examples of other-generated cues include wall posts by friends on the PO's wall and clicking "like” in response to a status update. An example of a system-generated cue includes the number of pictures uploaded.

Given that $\mathrm{HO}$ is a desirable trait, it is no surprise that a prior textual analysis of FB status updates (Carr et al. 2012) found that users expressed humor often. Carr et al. (2012) suggested that given the conversational nature of status updates, humor was a tactic for engaging friends. Status updates are just one self-generated cue of many possible cues on a FB profile. By analyzing self, other, and system generated cues the present investigation will offer a more comprehensive understanding of how humor is deployed on FB.

\subsection{The lens model}

The Brunswik (1956) lens model can help to answer questions of impression management and formation online. The purpose of a lens model analysis is to look at the relationship between perceptions of personality and actual (self-reported) personality traits through correlations with various possible communication interactions. This method allows researchers to ascertain what FB profile cues strangers use to estimate users' HO, what cues on users' profiles are actually associated with $\mathrm{HO}$, and the matching cues on a profile between strangers and POs. The lens model consists of three steps (Gifford 2006). In the first step, targets' (i.e., 
profile owners') FB profiles are gathered and their HO assessed. In the next step, independent coders content analyze the targets' profiles for a variety of cues (e.g., number of status updates, number of pictures posted). In the final step, observers, who are strangers to the targets, are asked to view all of the target profiles and estimate each target's HO. By evaluating what cues on a profile are correlated with targets' self-reported HO, the relationship between profile content and target disposition is determined. By evaluating the content associated with observers' estimates of targets' HO, the relationship between strangers' impressions of HO and FB profile construction can be ascertained. Finally, the cues that match between the two parts of the lens model, called functional achievement, can be identified. These cues are the aspects of a FB profile that are diagnostic of users' HO. Utilizing the full capacity of the lens model, we pose two research questions:

RQ1: What cues on a FB profile are associated with targets' HO?

RQ2: What cues on a FB profile are associated with observers' estimates of targets' $\mathrm{HO}$ ?

To better understand $\mathrm{HO}$ on FB, a complementary qualitative analysis of the profiles explored the dominant themes of humor on FB. This analysis was guided by the following question:

RQ3: What are the primary themes of humor use on FB?

\section{Method}

This study utilizes data gathered as part of a larger lens model analysis of $100 \mathrm{FB}$ profiles. This study required 35 observers to examine 100 targets' FB profiles, which included the main profile page, the 8 most recent profile pictures, recent wall posts/status updates, and the entire Info page of a profile. Observers estimated all 100 targets' HO by examining only their profile. Humor orientation was measured using the HO scale (Booth-Butterfield and Booth-Butterfield 1991) for targets' self-report and a modified version of the HO was used for observer estimates. A total of 53 cues from targets' FB profiles were content analyzed and independently coded by up to four coders. The qualitative phase of this study explored the results of the lens model analysis in greater depth. Qualitative analysis of text and pictures can provide an understanding of the data set that cannot be reached through the quantitative analysis alone (Lindlof and Taylor 2011). To achieve this goal, all target FB profiles were re-analyzed focusing on the cues showing functional achievement in the lens model analysis. 


\subsection{Profile owner targets}

One hundred targets were recruited through a combination of students at a large Midwestern university who participated for partial course credit $(n=28)$ and a snowball sample of FB users contacted by the researchers $(n=72)$. This was done to obtain a quota sample of profiles that represented the latest demographics of FB users regarding age and gender. The mean age for target participants was 32.3 $(S D=12.23$, range $=18-62)$, and female participants accounted for $57 \%$ of the sample. The majority of target participants were white $(88 \%)$, yet other race/ ethnicities were also represented: 5\% mixed race, 4\% Asian-American, 2\% African-American, and 1\% Latino/Hispanic. Targets provided written consent for access to their FB profile and to link the profile downloaded with their HO scores.

\subsubsection{Target measures and procedures}

Targets were also asked to complete an online survey regarding their HO (BoothButterfield and Booth-Butterfield 1991) as well as several demographic questions. All HO items were measured using a 7-point Likert-type scale. Samples items included, "I regularly tell jokes when in groups" and "People usually laugh when I tell jokes or funny stories”. This measure was reliable $(\alpha=.92)$.

Upon completion of the survey, targets provided the researchers access to their FB profile. Each profile included the 8 most recent profile pictures, all information available on the Info page of the profile, and any wall posts listed as "recent" by FB. Recent refers to any post that is above the "see more posts" button that appeared on a profile at the time of data collection (March 2010). If a user updated FB daily these posts were from the past week, but if they used FB weekly or monthly user older posts were included. These files were saved and combined to form one PDF for each target. All references to the targets' names were erased and replaced with "PO" (i.e., profile owner). In addition, any identifying information (i.e., contact information, email address, AIM screen name) was removed to ensure target participant confidentiality.

\subsection{Independent profile coding}

47 of the 53 cues were coded by up to four independent coders for all target profiles (codebook available upon request from study authors). Codes were broken 
into six larger categories of what is typically found for a FB profile with cues related to: the current profile picture, past profile pictures, wall activity, status updates, general text use, and the Info page. These categories were developed in consultation with recent online lens model research (e.g., Marcus et al. 2006) and past FB research (e.g., Walther et al. 2009; Utz, 2010), as well as from researchers' experience with FB. Coders were trained as a group by using a set of sample profiles not included in the study data set. The training period helped to develop the codebook and define the cues most often found on profiles. The training increased reliability between coders and clarified study measures. Reliability was measured using Krippendorf's alpha MACRO for SPSS (Hayes and Krippendorf 2007). Following training, each coder was asked to perform a content analysis all 100 profiles for some portion of the cues, a technique consistent with the lens model approach. Eleven cues that were measured using a yes/no question were coded by one rater. A random set of 25 profiles was later coded for all 11 yes/no codes by a second independent rater. Reliability estimates for 10 of 11 yes/no cues were 1.00 (profile picture at younger age $=.96$ ). Two coders were used for cues that involved counts (e.g., the number of likes in response to all status updates). Cues coded by 3 or 4 coders were rates or scales (e.g., Amount of political talk in status updates; Degree to which profile pictures attempted humor). The overall average reliability was .75, which is higher than a prior published lens model analysis coding a similar number of cues (Gosling et al. 2002).

The text from status updates was coded for the remaining 8 cues using Diction (Hart and Carol 2011), an automated linguistic analysis software program. This involved having each status update text copied and pasted into separate text files that were then run through the Diction program.

\subsection{Observer estimates}

Observers, who were strangers to the targets, examined the profile of each of the 100 targets and then estimated the HO of each target. HO estimates were made using the majority of target items, only presented in terms of how the observer saw the target (i.e., "This person regularly tells jokes when in groups"). Two items that could not translate (e.g., "I have no memory for jokes") were excluded. The reliability of the scale was high $(\alpha=.96)$. The evaluation of each target took between 8-15 minutes, resulting in a total of approximately 12-16 hours per person. To prevent fatigue, observers signed up for several smaller time blocks, and after 10 targets were evaluated, observers took a 10-minute break. Observers were paid \$75 upon completion. 
A total of 35 observers began the evaluation of FB profiles. Thirty observers completed all 100 targets, and 5 dropped out for varying reasons (e.g., time conflicts). These 5 observers evaluated between 6 and 33 profiles $(M=17.8)$. All observer ratings were included in the final analysis to strengthen the overall $\mathrm{HO}$ estimate for each target. ${ }^{1}$ Observers were primarily students at the same university as target participants $(n=32)$, but some were community members who responded to advertisements $(n=3)$. If observers knew a target personally, they were asked to skip and go to the next profile.

\subsection{Qualitative data analysis procedures}

The same 100 FB profiles used in the lens model analysis were examined in the thematic analysis conducted by the first author of this study. This analysis involved a close reading of the data for consistent categories (Creswell 2007). Each profile was read through and re-read through, producing a set of codes that were collapsed into eight categories. To better understand how the categories related to each other thematically, a matrix analysis was used to compare and contrast them. As explained by Miles and Huberman (1994), a matrix involves "the crossing or two or more main dimensions ... to see how they interact” (239). The creation of a matrix to establish themes and relationships creates a systematic approach to further understand the data, beyond what would be illustrated by content codes alone (Miles and Huberman 1994). Averill (2002) notes "matrices can be valuable agents in the search for relationships between/among categories of data" (856). As a result of the matrix and thematic analyses, the eight categories were collapsed into three themes that best captured the kinds of humor presented on a FB profile: life event oriented, pop-culture based, and self-related. Coupled with a thematic analysis, the matrix analysis gives added support for the conclusions of the qualitative analysis (Averill 2002).

1 Observers evaluated one FB profile at a time and then estimated HO based on the profile; they only proceeded to the next profile after completing each estimate. All observer reports for profiles were used because each was a complete observation of a profile, regardless of whether they analyzed all 100 profiles. If an observer was familiar with a person in the FB profile, they skipped that profile. By keeping people who did not complete all profiles, profiles that were skipped were supplemented by those who completed less than the total 100. Each observer estimate increased the number of observers assigned to each FB profile, so there was no reason to exclude their observations. While this creates some variance in the number of observers for each given profile, it increases the power to make claims with more analyses. 


\section{Results from the lens model analysis}

\subsection{Cue validity}

Correlations between the 53 cues and the targets' self-reported HO were calculated to determine which cues were valid indicators of targets' HO. These correlations are collectively referred to as cue validity (see Table 1, left-hand column; Gifford 2006). Targets who were high in HO significantly attempted humor in the three sections of a profile (pictures, status updates, and Info page). High HO targets also had significantly more relational talk in their status updates and had significantly more friendly and outgoing looking friends in their profile pictures. Correlations approaching significance were also found in regards to status updates that contained political talk (negatively), the number of "likes" by FB friends in response to a status update, the number of unique FB friend comments in response to status updates, and the total number of posts by the target. These results suggest that a user who is high in HO displays their ability to produce humor in all aspects of the profile, including profile pictures, status updates, and the Info page, and in doing so encourages likes and comments from FB friends.

\subsection{Cue utilization and functional achievement}

Correlations were also calculated between FB cues and observers' aggregate estimates of targets' HO. These correlations are referred to collectively as cue utilization correlations (see Table 1, right-hand column; Gifford 2006). Results suggest that observers rely upon many different cues to estimate targets' HO. Results also suggest that correlations between profile content and estimates made by observers are more strongly correlated than for cue validity, which is consistent with past lens model research on CMC (Marcus et al. 2006).

Several cues showed functional achievement (a match) between both sides of the lens model. The results suggest that cues relied upon by observers to estimate target $\mathrm{HO}$ were frequently the same cues indicative of HO. Other cues that matched on both sides included: attempts at humor in pictures and quotes, status updates that contained relational talk, number of FB friends' "likes" in response to status updates, the number of unique FB friends who commented on status updates, status updates that contained political talk (negatively), and having friendly and outgoing looking friends in profile pictures. Although the correlations were significant, the reliability of status updates in humor $(\alpha=.35)$ was very low, making it difficult to ascertain its unique role in understanding the ability to diagnose $\mathrm{HO}$ 
Table 1: Correlations between Self and Other Estimates of $\mathrm{HO}$ and Profile Cues

\begin{tabular}{|c|c|c|c|}
\hline \multicolumn{3}{|c|}{ Cue Validity (self-ratings) } & Cue Utilization (observers) \\
\hline Humor & $\#$ & Profile Cues & Humor \\
\hline & & Current Profile Picture & \\
\hline$-.16^{+}$ & 89 & Different Age & -.02 \\
\hline \multirow[t]{2}{*}{-.05} & 100 & Not of Person & -.13 \\
\hline & & Past 8 Profile Pictures & \\
\hline .13 & 90 & Friendliness & $.40^{\star \star \star}$ \\
\hline-.08 & 90 & Attractiveness & .09 \\
\hline $.21^{*}$ & 100 & Humor & $.43^{\star \star \star}$ \\
\hline .14 & 92 & Sociability & $.29^{\star *}$ \\
\hline $.16^{+}$ & 100 & Sexuality & .05 \\
\hline-.02 & 100 & Friends in Photos & $.27^{\star *}$ \\
\hline .02 & 86 & Friends' Attractiveness & $.21^{*}$ \\
\hline $.21^{*}$ & 87 & Friends’ Friendliness & $.40^{\star * \star}$ \\
\hline \multirow[t]{2}{*}{.13} & 100 & Drinking Occurring & $.29^{* *}$ \\
\hline & & Information Page & \\
\hline $.18^{+}$ & 100 & Movies Listed & .07 \\
\hline-.07 & 100 & Music Listed & -.07 \\
\hline-.09 & 100 & Books Listed & -.04 \\
\hline .02 & 100 & Games Listed & $-.19^{+}$ \\
\hline-.01 & 82 & Relationship Status & .00 \\
\hline .08 & 100 & Total \# Friends & $.27^{* *}$ \\
\hline .09 & 100 & Facebook Games & $-.21^{*}$ \\
\hline .08 & 100 & Pictures Posted & .03 \\
\hline .07 & 95 & Activeness of Profile Activities & $.22^{*}$ \\
\hline .13 & 100 & Total Quotes & .11 \\
\hline .07 & 100 & Religious Quotes & -.07 \\
\hline .06 & 100 & Literary Quotes & .09 \\
\hline $.16^{+}$ & 100 & Humor Quotes & $.21^{*}$ \\
\hline .04 & 100 & Inspirational Quotes & -.01 \\
\hline-.01 & 100 & Movie Quotes & .01 \\
\hline .09 & 100 & Song Quotes & .00 \\
\hline
\end{tabular}


Table 1 (cont.)

Cue Validity (self-ratings)

Cue Utilization (observers)

\begin{tabular}{|c|c|c|c|}
\hline Humor & $\#$ & Profile Cues & Humor \\
\hline & & Text Use & \\
\hline .14 & 84 & Total Words & .00 \\
\hline .13 & 84 & Total Characters & .00 \\
\hline$-.16^{+}$ & 84 & Average Word Size & -.06 \\
\hline $.15^{+}$ & 84 & \# Different Words & .01 \\
\hline .14 & 100 & Emoticon Use & -.07 \\
\hline .17 & 100 & Short-hand Use & $.18^{+}$ \\
\hline .14 & 100 & Laughter Use & .14 \\
\hline \multirow[t]{2}{*}{.10} & 100 & Extended Letter/Word Use & .06 \\
\hline & & Status Updates & \\
\hline .03 & 90 & Positive Affect & $.25^{*}$ \\
\hline .07 & 90 & Negative Affect & -.10 \\
\hline $.23^{*}$ & 90 & Humor & $.60^{\star \star \star}$ \\
\hline .04 & 90 & Fiscal Conservative & .14 \\
\hline$-.17^{+}$ & 90 & Political at All & $-.27^{\star \star}$ \\
\hline $.20^{*}$ & 90 & Relational Talk & $.20^{*}$ \\
\hline $.16^{+}$ & 90 & Family Talk & .04 \\
\hline .04 & 90 & Emotional Support & -.13 \\
\hline-.00 & 90 & Instrumental Support & -.07 \\
\hline-.05 & 90 & Religious Quotes & -.08 \\
\hline .27 & 11 & Religiousness & -.29 \\
\hline .03 & 92 & Media Posts & $-.22^{*}$ \\
\hline .07 & 92 & Music Posts & .00 \\
\hline \multirow[t]{2}{*}{-.02} & 92 & News Posts & $-.16^{+}$ \\
\hline & & Wall Activity & \\
\hline .03 & 100 & Comment's on Other's Posts & .10 \\
\hline $.17^{+}$ & 100 & Total \# Posts & -.08 \\
\hline-.01 & 100 & Friends Agreement with Posts & -.08 \\
\hline $.17^{+}$ & 100 & \# Likes on Status Updates & $.28^{* *}$ \\
\hline $.17^{+}$ & 100 & \# Unique Comments & $.23^{*}$ \\
\hline \multicolumn{4}{|c|}{ Note: $+p<.10 ;{ }^{\star} p<.05 ;{ }^{\star \star} p<.01{ }^{\star \star \star} p<.001$} \\
\hline
\end{tabular}


online. The subsequent qualitative analysis of humor through profiles sought to clarify the role of status updates further.

In addition to cues that showed functional achievement, observer estimates of $\mathrm{HO}$ were significantly correlated with other cues associated with profile pictures: the friendliness of the target, the social activities of targets, are they drinking alcohol, and the total number of friends present. Observers also used information from status updates and wall posts to estimate HO; perceiving that the expression of positive affect in updates, status updates that contained media posts (negatively), total number of FB friends, and FB games played (negatively) were related to targets' HO. While these cues did not show functional achievement, and thus, are not diagnostic of HO, they do point to information that observers use to estimate targets' $\mathrm{HO}$ that users ought to be aware of, as they can be sources of misperception of $\mathrm{HO}$ on FB.

When estimating targets' HO, status updates were most often used, followed by images, and finally other-generated cues (e.g., friends' likes). Self-generated cues were more diagnostic of targets' HO, with only two other-generated cues finding agreement from both self-report and observer estimates. No systemgenerated cues showed functional achievement for $\mathrm{HO}$, which suggests that system-generated information on FB is not useful in forming impressions of HO.

\subsection{Overall accuracy}

The lens model can determine the overall accuracy with which the observers estimate the targets' HO (Gifford 2006). To calculate accuracy, self-reported HO by the target was correlated with all observers' aggregate estimate of the target's HO. The mean accuracy score was $r=.22, p<.01$, and comparable with past lens model accuracy for judging the Big Five personality traits (Gosling et al. 2002; Marcus et al. 2006). These results suggest that $\mathrm{HO}$ can be accurately judged by only viewing a FB profile for 10-15 minutes, and can be judged with similar overall levels of accuracy as personality.

\section{Results from the qualitative analysis}

\subsection{Life event oriented humor}

The qualitative analysis revealed that daily life events were the primary focus of humor on FB. These humorous remarks were most often expressed through status 
updates. The topic of daily events ranged from encounters at work or at home, to spending time with friends and loved ones. In an example of a work-related joke, one participant, who was a teacher, posted the following: "Quizzes - natural grade deflation," which received 6 likes from the user's FB friends. In an example of a post about domestic life, one user posts: "There is NOTHING stickier, more durable, and harder to scrape away than day-old fruity pebbles ... they should build the next space shuttle out of them", which received over 10 likes from the user's FB friends. It is worth noting that status updates that contained life event oriented humor generally tended to receive more "likes" from FB friends in comparison to other status updates (e.g., political posts, music posts). This helps to explain why the number of "likes" correlated with targets' HO, and why more frequent status updates containing political talk would be negatively related to HO.

The quantitative analysis suggested that users with more status updates containing relational talk were considered more humorous. The thematic qualitative analysis revealed that life event oriented humor often intersected with relational talk. Examples of status updates containing both humor and relational talk included: "I'm cutting so many people from my friends list, I might not have a newsfeed when I'm done”, “OMG, Joe is drinking landshark, does this mean I have to take him on my vacations with me? Haha", and "Just made wicked awesome coconut curry chicken, but I may have killed my acid reflux-suffering boyfriend in the process". These personal stories also tended to receive more "likes" from FB friends.

Daily life humor sometimes appeared in quotes in the Info page as well. Jokes in the quotes section were often presented as snippets of dialogue from past events, what might best referred to as "inside jokes". For example, one user lists the following as a favorite quote: "Tara and I in line at McD at 4am w/ my puppy Capt Steve; Tara: 'So we're getting 4 double cheeseburgers?' Steve: 'Arrrf!' Tara: ' 5 it is!'” Daily life events was the one type of humor that was primarily textbased, while humor displayed in images can be seen more prominently in the themes discussed below.

\subsection{Pop-culture based humor}

Popular culture references abound on FB. Whether in status updates, humor quotes, or even pictures, participants discussed current events made popular in recent months, often through celebrities. As previously noted, while the reliability of status updates containing humor was low, further analysis of this cue in the qualitative section clarifies its role in online humor production. Status updates 
that contained humor that was specifically pop-culture based tended to get a higher number of "likes" from FB friends, showing a relationship between these two cues. One common example of this theme were references to Charlie Sheen, who famously went through a very public break from his TV show Two and a Half Men in the spring of 2010 (the time period during which data were gathered). Status updates and quotes on the Info page often appropriated his claim that he was "winning" in attempts to show humor. Another popular show, Saturday Night Live (SNL) appeared in target profiles frequently ranging from links to SNL clips to this example from a status update that proclaimed, "I was watching SNL tonight and thought Miley Cyrus was hosting, but it turns out it was just Hannah Montana”. Other status updates that referenced celebrities reflected issues germane to FB itself. One status update joked, "lil wayne may have dropped the soap, but I'm not clicking that link to find out ...”. In this status update, the user makes light of people getting a computer virus by following a link on FB that is disguised as a popular culture story.

Popular culture references were repeated in various locations on users' profiles in a manner that often reflected these users' personal interests. Consider here the user who joked about Miley Cyrus in a status update also had a quote on their Info page about her from Sean "P. Diddy" Combs: "That is some adult Hannah Montana shit". Users photo images also came into play: some users had profile pictures that mimicked celebrities and other famous figures in popular culture.

\subsection{Self-related humor}

A final popular subject for humor deployed on FB was humor directed towards and related to the self. This type of humor was found more often in status updates and photos, such as a silly pose or face, than on the quotes portion of the Info page. Many POs that rated high in $\mathrm{HO}$ had had at some point a profile picture that showed the PO making fun of himself or herself. One example of humor in pictures would be silly Halloween costumes.

Other self-directed humor included humorous statements that were thematically about the PO and his or her own personality or experiences. Interestingly, self-related humor on status updates that was not self-deprecating tended to receive a high number of likes, but self-related updates that were humorous but self-deprecating tended to receive more comments than likes. Examples of humor used in status updates that were self-related but not necessarily self-deprecating include: "Lost to a [debate] team today whose last words were 'to uphold the 
law would be to allow a man to marry his house' I'm sure that gem of wisdom was worth a near perfect score", and, referring to another FB friend, "Flossy McHookerpants? Man my name is * $\mathrm{so}^{\star}$ boring”. In each instance, the self or a personal experience is part of the joke, but the humor is predicated on making fun of someone else (mocking the debate team the FB user lost to, or joking about their own name being boring in relation to a nickname of a FB friend), rather than suggesting they themselves are at fault.

By comparison, self-deprecating humor used in status updates tended to receive comments rather than likes from FB friends. Examples of these updates included: " $<$ heart> I figured out why I'm fat! The shampoo I use in the shower that runs down my body says 'for extra volume and body' I'm going to start using 'Dawn' dish soap. It says 'dissolves fat that is otherwise difficult to remove.'

${ }^{\star \star}$ Repost if you have the same issue. <heart>”, "Changing my relationship status to out of order", "So Sarah and I were just discussing how we need to be more financially responsible - then we went shopping. Crap! Gets me everytime!!!”, and "I should REALLY be shopping a ball gown, and NO not because someone mistook me for a high school student and invited me to prom ...”. In these cases, the self-deprecating humor made fun of the user for a variety of reasons (weight, youthful appearance). Interestingly, these self-disparaging comments generated responses from FB friends that typically negated the claims. In doing so, FB friends tended to show support or tried to include themselves in the joke. Sometimes the humorous status update was carried on and developed through comments by the user and their FB friends creating witty back-and-forth banter.

\section{Discussion}

The results of the present investigation show that humor is engrained in how users engage with FB as they construct a profile of self and interact with friends and strangers alike. These results demonstrate a desire by users to convey a humorous persona through their FB profile as a way to build and maintain social connections, and to cope with stress experienced on a daily basis. When a user is stressed they will write about it in a way to entertain FB friends and shows the user is capable of producing humor, which helps to alleviate that stress. The cues found to be diagnostic suggest that strangers can accurately form impressions of users' HO by using a variety of FB cues. Important to note is that the overall accuracy with which strangers estimated users' HO suggests that strangers can accurately estimate a users' HO by only examining their FB profile for 10-15 minutes, which can be integral to future research on first impressions. The specific 
cues that are indicative of HO and the themes of humor expression on FB are discussed in greater detail below.

\subsection{Accurately perceiving $\mathrm{HO}$ on FB}

The results of the lens model analyses indicated that FB users who self-reported a higher HO were more likely to attempt humor across all sections of a FB profile on multiple topics. As Booth-Butterfield and Booth-Butterfield (1991) point out, those with a high $\mathrm{HO}$ attempt humor in various contexts and make repeated efforts to try to crack jokes, even in the face of failure. The results of this study offer clear evidence of the utility of the HO measure in online environments, indicating that, similar to past research on various personality traits, our online behaviors often mimic our offline behaviors. Furthermore, observers, who were strangers to the POs, used all three of these cues (i.e., humor in status updates, pictures, quotes) to accurately estimate profile owners' HO. It is important to note that the independent coders performing the content analysis merely quantified the attempts at humor, not how subjectively or objectively humorous the status updates, quotes, or pictures were. This means that simply attempting more humor in all three sections of the FB profile is both related to being a funny person and is effective at conveying the impression that one is funny.

There were several other FB profile cues that were diagnostic of users' humorous disposition and used by strangers to estimate HO: talking about relationships in status updates, the number of FB friends who "liked" status updates, the number of unique FB friends commenting on status updates, having more friendly and outgoing friends in profile pictures. The thematic analysis conducted underscores why more relationships talk and less political talk was indicative of a profile owners' HO: impersonal political status updates tended to be more serious and less playful than other topics of status updates, hence the negative correlation. Indeed, the thematic analysis revealed that the most common attempts at humor on FB were personal life event humor. Personal anecdotes are a common topic of humor in face-to-face conversations as well (Hay 2001). Status updates about personal relationships often convey a bit of humor or irony to elicit amusement from one's FB audience.

The lens model analysis also showed that both the number of "likes" and the number of unique friends' comments in response to users' status updates were indicative of a more humorous disposition and were used to estimate targets' HO. Thematic qualitative analyses further developed this finding by demonstrating that FB friends' "likes" and comments reflected the expression of two different 
styles of humor. Given the significant correlation between number of "likes" on a status update and those updates that contained humor in some form, results suggest that expressing an anecdotal and innocuous style of humor on updates was well received by one's social network. This supports past literature on humor in fan forums that suggested that online community members are most interested in reading posts that contain humor (Baym 1995).

Interestingly, the results of the present investigation suggest that selfdepreciating humor generates more comments than "likes" from FB friends. This is reasonable given that a FB "like" is often viewed as agreement or approval with that statement. Past conversation analysis has shown that self-deprecating humor is generally followed up with support or sympathy from friends (Hay 2001). On FB, self-deprecating status updates tend to generate support or further selfdeprecating joking through comments by friends who negate or humorously identify with the update the user posted, showing a relationship between offline and online behaviors.

In comparison to past analyses of textual humor use on FB (Carr et al. 2012) the present study offered a more detailed analysis of multitude of cues available on FB. Consequently, the results contribute to an ongoing discussion of the warranting value of self, other, and system-generated cues (Walther et al. 2009; Utz 2010). Specifically, past research suggests that other-generated cues are useful when strangers wish to form an accurate impression of a FB user (Walther et al. 2009). However, the results of the present investigation suggest that it is a combination of self and a few crucial other-generated cues on FB profiles that are diagnostic of the profile owner's HO. This may be due to something unique to humor. While repeated attempts at humor by the FB user - regardless of audience response - conveyed a humorous impression, the responses of users' FB friends appear to bolster or warrant the users' funny self-presentation.

\subsection{Misperceiving a humorous disposition on FB}

There were several cues that observers relied on to estimate HO that were not diagnostic of a users' HO. These cues are not to be discounted, but, rather, are important to consider in relation to each other and what they mean for how HO is perceived online. Given the cue utilization correlations that were not diagnostic matches, it appears that strangers believe that an outgoing individual with many FB friends is indicative of possessing HO. Strangers appeared to rely particularly on profile pictures when forming an impression of users' HO. Given humor's relationship with social attractiveness and skill (Merolla 2006), it makes sense that 
strangers would use these cues to judge users' HO. POs who had more friends in pictures and more attractive friends, who had photos in a social environment or while drinking were perceived by observers to have a higher HO. Only one diagnostic cue existed in pictures outside of the overt use of humor: the friendliness of friends in profile pictures. Consistent with past lens model studies (Gifford 2006), observers employ a variety of intuitive cues to form judgments, but simply because a cue is intuitive does not make it diagnostic or lead to more accurate impressions. Knowing that these misperceptions exist is the first step in avoiding an inaccurate assessment of an individual's personality.

\subsection{Themes of humor use}

The various themes of humor expressed throughout FB profiles demonstrate that humor is used in a variety of contexts and for many reasons online much as it is offline (Martin 1998; Wanzer et al. 2009). This is consistent with past research, which suggested that a common feature of online humor is a focus on celebrities (Shifman 2007). We also see how humor is displayed as a form of social sharing and impression management similar to technologies discussed earlier (Hübler and Bell 2003). There was also frequent overlap in themes. A status update could be about something a user did and also include the hashtag \#winning at the end, in reference to an online comment by Charlie Sheen. ${ }^{2}$ This example would encompass all three of the primary themes of humor on FB (i.e., pop culture, self, and daily events). This points to the role of status updates and norms that have developed on FB to focus on certain topics of discussion.

This analysis also demonstrates how humor can be used as a coping mechanism, regardless of whether the attempt is successful at producing a humorous effect (Martin 1998). Consider the earlier example of the PO complaining about losing to another debate team. In this status, the user engaged in self-defeating humor by admitting their loss, life-event based humor by talking about something that happened during their day, but also some attacking humor because the overall message made fun of the team who beat them. The status update appeared to generate both amusement and sympathy from friends, providing an outlet to build connections through the production of humor that is unique to FB.

2 In 2010 Charlie Sheen began to post online about how great his life was after quitting the hit show Two and a Half Men, using the hashtag "\#winning” to indicate he was better off without the show. This became a running joke used by people across social media. 


\subsection{Limitations}

Given the evolving nature of FB, new cues may arise as old cues disappear. Already the addition of Timeline has changed how the profile is displayed. Programs that write status updates for a PO (i.e., Status Shuffle) now exist, which could alter perceptions of users. Although it is unlikely that these programs had an effect on the evaluation of HO by observers in the present investigation, in the future it is important to account for cues that were once self-generated but could now be system-generated. Furthermore, although our study used a quota sample, it was a non-probability sample, which can diminish the generalizability of claims. Finally, codes with low reliabilities on the cue validity or cue utilization sides may have resulted in a failure to detect other significant relationships.

In conclusion, this study confirms past research suggesting humor plays a key role in our everyday lives and interactions (Martin 1998), even online. It also validates claims that online identity claims are extensions of our offline self, which is important for future research on interpersonal communication and new media. The frequent use of humor on FB also supports previous research on how humor can be deployed on SNS (e.g., Carr et al. 2012), and supports the idea that the goal of a FB status update or quote is not just to inform an audience, but also to entertain. This is important in a continued line of research on how self is constructed online and the accuracy of perceptions formed based on available cues. Altogether, these results provide a cohesive image of how humor is conveyed and perceived in one of the most popular online environments, FB, which can allow users of the site to more accurately assess this important personality trait.

\section{References}

Averill, Jennifer B. 2002. Matrix analysis as a complementary analytic strategy in qualitative inquiry. Qualitative Health Research 12(6). 855-866.

Baym, Nancy. 1995. The performance of humor in computer-mediated communication. Journal of Computer-Mediated Communication 1(2). doi: 10.1111/j.1083-6101.1995.tb00327.x.

Booth-Butterfield, Steve \& Melanie Booth-Butterfield. 1991. The communication of humor in everyday life: The use of humorous messages. Southern Communication Journal 56. 205-218.

Brunswik, Egon. 1956. Perception and the representative design of psychological experiments. Berkeley, CA: University of California Press.

Cann, Arnie, Lawrence G. Calhoun, \& Janet S. Bank. 1997. On the role of humor appreciation in interpersonal attraction: It's no joking matter. Humor: International Journal of Humor Research 10(1). 77-89. 
Carr, Caleb T., David B. Schrock \& Patricia Dauterman. 2012. Speech act analysis within Social Network Sites' status messages. Journal of Language and Social Psychology 31(2). 176-196.

Craik, Kenneth H. \& Aaron P. Ware. 1998. Humor and personality in everyday life. In Willibald Ruch (ed.), The sense of humor: Explorations of a personality characteristic, 63-94. Berlin \& New York: Mouton de Gruyter.

Cresswell, John W. 2007. Qualitative inquiry \& research design: Choosing among five approaches. Thousand Oaks, CA: Sage.

Facebook Statistics. 2011. http://www.new.facebook.com/friends/\#/press/info.php?statistics (accessed on 18 December 2011).

Gifford, Robert. 2006. Personality and nonverbal behavior: A complex conundrum. In Valerie Manusov \& Miles L. Patterson (eds.), Handbook of nonverbal communication, 159-180. Thousand Oaks, CA: Sage.

Goffman, Erving. 1959. The presentation of self in everyday life. New York, NY: Anchor Books.

Gosling, Samuel D., Sei J. Ko, Thomas Mannarelli \& Margaret E. Morris. 2002. Room with a cue: Personality judgments based on offices and bedrooms. Journal of Personality and Social Psychology 82(3). 379-398.

Hart, Rodrick P. \& Craig Carroll. 2011. DICTION 6.0 [Computer software]. http://www. dictionsoftware.com/index.php (accessed on 26 October 2011).

Hay, Jennifer. 2001. The pragmatics of humor support. Humor: International Journal of Humor Research 14(1). 55-82.

Hayes, Andrew F. \& Klaus Krippendorf. 2007. Answering the call for a standard reliability measure for coding data. Communication Methods and Measures 1(1). 77-89.

Hübler, Mike T. \& Diana C. Bell. 2003. Computer-mediated humor and ethos: Exploring threads of constitutive laughter in online communities. Computers and Composition 20(3). 277-294.

Lindlof, Thomas R. \& Bryan C. Taylor. 2011. Qualitative communication research methods, 3rd edn. Thousand Oaks, CA: Sage.

Marcus, Bernd, Franz Machilek \& Astrid Schütz. 2006. Personality in cyberspace: Personal websites as media for personality expressions and impressions. Journal of Personality and Social Psychology 90(6). 1014-1031.

Martin, Rod A. 1998. Approaches to the sense of humor: A historical review. In Willibald Ruch (ed.), The sense of humor: Explorations of a personality characteristic. 15-60. Berlin \& New York: Mouton de Gruyter.

Merolla, Andy J. 2006. Decoding ability and humor production. Communication Quarterly 54(2). 175-189.

Miles, Matthew B. \& A. Michael Huberman. 1994. Qualitative data analysis, 2nd edn. Thousand Oaks, CA: Sage.

Nastri, Jacqueline, Jorge Peña \& Jeffrey T. Hancock. 2006. The construction of away messages: A speech act analysis. Journal of Computer-Mediated Communication 11(4). http://jcmc. indiana.edu/vol11/issue4/nastri.html (accessed 14 November 2011).

Rains, Stephanie A., Geoffrey R. Tumlin \& Mark L. Knapp. 2009. Electronic bumper stickers: The content and interpersonal functions of messages attached to e-mail signatures. Discourse Studies 11(1). 105-121.

Shifman, Limor. 2007. Humor in the age of digital reproduction: continuity and change in Internet-based comic texts. International Journal of Communication 1. 187-209.

Utz, Sonja. 2010. Show me your friends and I will tell you what type of a person you are: How one's profile, number of friends, and type of friends influence impression formation on SNS. Journal of Computer-Mediated Communication 15(2). 314-335. 
Walther, Joseph B. 1996. Computer-mediated communication: Impersonal, interpersonal, and hyperpersonal interaction. Communication Research 23(1). 3-43.

Walther, Joseph B., Brandon van der Heide, Lauren M. Hamel \& Hillary C. Shulman. 2009. Self-generated versus other-generated statements and impressions in computer-mediated communication: A test of warranting theory using Facebook. Communication Research 36(2). 229-253.

Wanzer, Melissa B., Melanie Booth-Butterfield \& Steve Booth-Butterfield. 1996. Are funny people popular? An examination of humor orientation, loneliness, and social attraction. Communication Quarterly 44(1). 42-53.

Wilbur, Christopher J. \& Lorne Campbell. 2011. Humor in romantic contexts: Do men participate and women evaluate? Personality and Social Psychology Bulletin 37(7). 918-929.

Zywica, Jolene \& James Danowski. 2008. The faces of Facebookers: Investigating social enhancement and social compensation hypotheses. Journal of Computer-Mediated Communication 14(1). 1-34.

\section{Bionotes}

Natalie Pennington (MA Kansas State University) is a doctoral candidate in the Department of Communication Studies at The University of Kansas. Her research focuses on interpersonal communication online through social media, and more specifically, impression management, social support, and relational development. E-mail: natpen@ku.edu

Jeffrey A. Hall (PhD University of Southern California) is an associate professor in the Department of Communication Studies at The University of Kansas. His research focuses on dating and flirting, friendship, online communication, humor in long term relationships, and homophobia and masculinity. E-mail: hallj@ku.edu 